\title{
Breath figure templated self-assembly of surface-acylated cellulose nanowhiskers confined as honeycomb films
}

\author{
Huan Liu • Bo Pang $\cdot$ Kai Zhang
}

Received: 15 July 2021 / Accepted: 30 September 2021 / Published online: 13 October 2021

(C) The Author(s) 2021

\begin{abstract}
The self-assembly of cellulose nanowhiskers (CNWs) in confined geometries provides a powerful method for the fabrication of novel structures. Herein, ordered honeycomb microporous films were first prepared with surface-acylated CNWs (CNWs-SU) through the breath figure method. Resulting films showed highly porous order over large regions and the iridescent color was only displayed by their rims, which is different from traditional dish-cast CNW films showing the iridescent color over the whole area. This is mainly due to the condensation of water droplets forming three-dimensional (3D) geometry, which forced CNWs-SU to self-assemble into cholesteric architectures in confined geometry and resulted in the iridescent color of the rims after drying. The mechanism was further studied by investigating the critical influencing factors, primarily the
\end{abstract}

Supplementary Information The online version contains supplementary material available at https://doi.org/10.1007/ s10570-021-04245-3.

\section{H. Liu}

Biofuels Institute, School of the Environment and Safety Engineering, Jiangsu University, No. 301 Xuefu Road, Zhenjiang 212013, China

H. Liu $\cdot$ B. Pang $\cdot$ K. Zhang $(\bowtie)$

Sustainable Materials and Chemistry, Department of Wood Technology and Wood-Based Composites, GeorgAugust-University of Goettingen, Büsgenweg 4, 37077 Göttingen, Germany

e-mail: kai.zhang@uni-goettingen.de concentration of CNW-SU suspensions, the relative humidity of the atmosphere and the surface-attached moieties. In particular, CNW-SU suspensions with a concentration of $3 \mathrm{mg} / \mathrm{mL}$ at the relative humidity of $75 \%$ preferentially formed honeycomb films with uniform pores. Too low or too high concentrations of CNW-SU suspensions or relative humidity are not preferable for uniform porous films. CNWs-SU with further immobilized octadecane or fluoroalkyl groups on their surface strongly affected the formation of uniform porous films because of higher hydrophobicity and accompanying inhomogeneous condensation of water droplets. This work provides a novel method to study the interactions of CNWs beyond the planar geometry and the formation of uniform porous films solely with CNWs with structural colors open up interesting possibilities for broad application in photonic nanomaterials. 


\section{Graphic abstract}

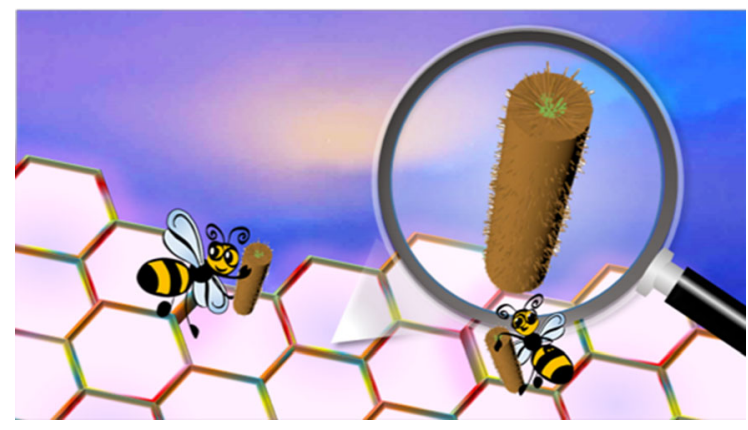

Keywords Breath figure - Self-assembly $\cdot$ Cellulose nanowhiskers $\cdot$ Honeycomb film

\section{Introduction}

The self-assembly of CNWs has attracted intensive interest in the nanoscience and materials science community, owing to their ability to form chiral nematic structures in aqueous suspensions (Bondeson et al. 2006; Habibi et al. 2010). Traditionally, the selfassembly of CNWs has been studied in planar geometry (Adstedt et al. 2020; Dumanli et al. 2014; Hiratani et al. 2017; Zhang et al. 2020). This is the simplest and most studied case of the chiral organization of CNWs in confined geometry, in which CNW suspensions are placed on substrates with the other surface being in direct contact with the atmosphere. Following the evaporation of water, CNWs can retain their chiral nematic order in resulting thin iridescent films, which are of interest for many applications, such as photonic sensors, filters, and reflectors or photoelectronic devices (Giese and Spengler 2019; Hiratani et al. 2017; Mahpeykar et al. 2017; Yao et al. 2017). More recently, many studies about the self-assembly of CNWs in two-dimensional or 3D confined geometries also emerged, since the confinement effect can effectively alter the symmetry of a structure and lead to strong deviation from an equilibrium morphology and thus resulting in the construction of novel structures ( $\mathrm{Li}$ et al. 2016). For the two-dimensional confinement, CNWs self-assemble in a continuous cylindrical geometry resulted in hierarchical liquid metacrystal fibers, which exhibit controllability on the polarized light-direction and -intensity (Liu and $\mathrm{Wu}$ 2020). The self-assembly of CNWs in two-dimensional capillary confinement was also investigated (Cherpak et al. 2018). The results demonstrated that the anisotropic drying and unidirectional propagation of the anisotropic phase in large areas were mainly induced by the saturated water vapor in one end of the capillary, resulting in the formation of chiral $\mathrm{CNW}$ films with uniformly oriented layered structures. Fully enclosed spherical geometry, as a kind of 3D confinement, has been applied to the self-assembly of CNWs (Li et al. 2016; Parker et al. 2016). Uniform spheres with chiral nematic structures from CNWs can be fabricated with this method. Although much progress has been reported about the self-assembly of CNWs in simple symmetric confined geometries, the selfassembly in more complicated confined geometries is still extremely challenging and rarely reported.

Honeycomb films with well-ordered pores with a narrow size distribution are of significant interest in advanced functional materials, such as separation (Du et al. 2013), templates (Galeotti et al. 2011), optical and optoelectronic devices (Yabu and Shimomura 2005), biosensors and biomaterials (Min et al. 2008; Wan et al. 2012), protein arrays (Ju et al. 2019), and cell culture scalfolds (Neznalová et al. 2020). Over the past few decades, a variety of techniques, including lithography (Kim et al. 2009), colloidal templates (Hu et al. 2009), emulsion (Kasai and Kondo 2004), improved phase separation (IPS) (Bui et al. 2015; Slepička et al. 2020), and breath figure (BF) (Huang et al. 2020), have been developed to create ordered structures with uniform pore sizes in the nano- to micrometer range. Among these approaches, BF has become the most attractive self-assembly technique for fabricating orderly packed pores with hexagonal array due to its simplicity and versatility (Bunz 2006; Zhang et al. 2015). Various types of synthetic polymers, including star, linear, and block copolymers, as well as polymer-particle systems, and semisynthetic biopolymers, are the most common raw materials to fabricate honeycomb films with controlled pore size using this technique (Zhang et al. 2015). Cellulose as a kind of biosynthesized and biodegradable polymer has been successful in obtaining porous films by BF method. Nemoto et al. (Nemoto et al. 2005) reported the fabrication of honeycomb films based on cellulose acetate. In this 
study, the acetate films were extremely irregular, having microporous sizes ranging from 1 to $100 \mu \mathrm{m}$.

Although honeycomb films are usually prepared from polymer solutions, other compounds that can stabilize the water droplets and form a continuous film can be used to replace polymer, producing nonpolymeric honeycomb films (Bai et al. 2013). Various nanomaterials, such as many kinds of metal and metal oxide nanoparticles (Sakatani et al. 2008), carbon nanotubes (Wakamatsu et al. 2009), and graphene oxide (Lee et al. 2010) have been widely exploited to fabricate honeycomb films due to their unique catalytic, photonic, and electric properties. The introduction of ordered patterns into these nanomaterials usually enhances their inherent properties and leads to new functions. Most of these nanomaterials have to be decorated by organic ligands or polymers, or with the assistance of surfactants, to achieve good dispersibility in organic solvents and adequate polarity for nanoparticles adsorption at the water/solvent interfaces to prevent water droplet coalescence during evaporation. However, direct use of polysaccharidederived nanomaterials including CNWs as raw materials for the fabrication of honeycomb films has not been reported. Moreover, to the best of our knowledge, the self-assembly of CNWs in a complicated confined geometry formed by the condensation of water droplets during the breath figure process has not been reported.

In this study, we investigated the self-assembly of surface-acylated hydrophobic CNWs (CNWs-SU) in confined spaces during the breath figure process, which led to highly ordered honeycomb films. To the best of our knowledge, this is the first example describing the formation of self-assembled honeycomb-patterned structures solely from nanocellulose. The resulting films show high porous order over large areas. Moreover, the rims of the obtained honeycomb film displayed iridescent colors due to the chiral nematic architectures formed by the self-assembly of CNWs-SU in the confined 3D walls of pores generated by the gradual condensation of water droplets. In addition, the effects of the concentration of the CNWSU suspensions, the relative humidity of the atmosphere, and the surface-attached moieties of CNWs on the self-assembly were also investigated.

\section{Experimental section}

Materials

Microcrystalline cellulose (Avicel PH101) with an average particle size of about $50 \mu \mathrm{m}$, and 10-undecenoyl chloride were purchased from Sigma-Aldrich Chemie GmbH (Steinheim, Germany). Dry pyridine and tetrahydrofuran (THF) were brought from Th. Geyer GmbH \& Co. KG (Renningen, Germany). All chemicals are all of the analytical grade and used as received. Deionized water was used in all experiments.

Preparation of surface-acylated cellulose nanowhiskers (CNWs-SU)

CNWs-SU were prepared according to our previous work with a few modifications (Liu et al. 2018). Typically, $1 \mathrm{~g}$ of microcrystalline cellulose and $30 \mathrm{~mL}$ of pyridine were fed into a three-necked flask. The reaction mixture was stirred and heated up to $50{ }^{\circ} \mathrm{C}$. Then, 10 -undecenoyl chloride $(2.73 \mathrm{~mL}, 2 \mathrm{~mol}$ acid chloride per mol anhydroglucose units of cellulose) was added to the cellulose suspension under stirring. After $7 \mathrm{~h}$ stirring at $50{ }^{\circ} \mathrm{C}$, the reaction mixture was poured into $200 \mathrm{~mL}$ methanol immediately. The precipitate was separated by centrifugation. Thereafter, the product was purified by a repeated dispersion and precipitation process using THF and methanol, respectively, before the product was dispersed in THF. Then, the suspension was centrifuged for $10 \mathrm{~h}$ at $14,000 \mathrm{rpm}$ at $4{ }^{\circ} \mathrm{C}$. After the centrifugation, the supernatant was removed and the solid was dispersed in THF again, which was centrifuged for $20 \mathrm{~min}$ at $3000 \mathrm{rpm}$ at $20{ }^{\circ} \mathrm{C}$ to remove the microscaled segments. Finally, the purified product was dispersed in DCM for further use. The corresponding yield of CNWs-SU was $11.6 \pm 2.4 \%$.

Post-modification of CNWs-SU via thiol-ene reaction with 1-octadecanethiol or $1 \mathrm{H}, 1 \mathrm{H}, 2 \mathrm{H}, 2 \mathrm{H}$ perfluorodecanethiol to synthesize CNWs-SU-C18 or CNWs-SU-F

1-octadecanethiol or $1 \mathrm{H}, 1 \mathrm{H}, 2 \mathrm{H}, 2 \mathrm{H}$-perfluorodecanethiol ( $0.75 \mathrm{~mol}$ per mol $\mathrm{C}=\mathrm{C}$ double bonds) was added into THF-suspension of CNW-SU (10 mg/ $\mathrm{mL})$. The mixture was exposed to UV light 
(320-400 nm with the intensity of $\sim 15 \mathrm{~mW} / \mathrm{cm}$ ) for $3 \mathrm{~h}$ at room temperature under stirring. After the reaction, all mixtures were transferred in dialysis membrane (with a molecular weight cut-off of $3500 \mathrm{Da}$ ) and kept in 5 volumes of THF for $12 \mathrm{~h}$. Then, THF was changed twice after every $12 \mathrm{~h}$. Thereafter, the dialyzed products were centrifuged for $20 \mathrm{~min}$ at $3000 \mathrm{rpm}$ at $20{ }^{\circ} \mathrm{C}$ to remove the aggregates. Finally, all purified CNWs-SU-C18 or CNWsSU-F were dispersed in dichloromethane (DCM) for further use. Based on the elemental analysis, the contents of octadecane and fluoroalkyl groups in CNWs-SU-C18 and CNWs-SU-F were determined to be 1.37 and $1.33 \mathrm{mmol} / \mathrm{g}$, respectively.

Preparation of honeycomb films from CNWs-SU, CNWs-SU-C18 and CNWs-SU-F

Honeycomb films with controlled microporous structures were fabricated via the breath figure method, as illustrated in Fig. S1. In a typical process, CNWs-SU, CNWs-SU-C18 or CNWs-SU-F were first dissolved in $\mathrm{DCM}$ to a certain concentration, and then $10 \mathrm{~mL}$ of the CNW-SU, CNWs-SU-C18 or CNWs-SU-F suspension was cast onto the Teflon mould in the desiccator at different relative humidity. The saturated relative humidity in the chamber was fixed by saturated salt solutions, i.e., $44 \%\left(\mathrm{~K}_{2} \mathrm{CO}_{3}\right), 57 \%(\mathrm{NaBr}), 75 \%$ $(\mathrm{NaCl})$, and $86 \%(\mathrm{KCl})$ (Young 1967). The temperature was kept at room temperature during the whole process. After the complete evaporation (about $10 \mathrm{~h}$ ) of solvent as well as the water droplets that spontaneously condensed at the air-suspension interface, honeycomb films with controlled porous structures were obtained on the surface of the Teflon substrate.

Characterization and measurement

\section{Transmission electron microscopy (TEM)}

TEM images of the CNWs-SU were acquired with a CM 12 Transmission Electron Microscope (Philips, Netherland). The specimen was stained by phosphotungstic acid solution ( $0.2 \mathrm{wt} \%$ in THF) for about 2 min to enhance the contrast.
Solid-state ${ }^{13}$ C CP-MAS NMR spectroscopy

Solid-state ${ }^{13} \mathrm{C}$ CP-MAS NMR measurements for all samples were acquired at ambient conditions using a Bruker AVANCE IIIHD 800WB spectrometer with a field strength of $18.8 \mathrm{~T}$. Experiments were performed using a Bruker $1.3 \mathrm{~mm}$ MAS triple resonance probe with a MAS spinning frequency of $55.555 \mathrm{kHz}$. A ramped CP-MAS sequence was used with a ${ }^{1} \mathrm{H}$ excitation power of $138.8 \mathrm{kHz}$ and a Hartman Hahn contact time of $2 \mathrm{~ms}$ with a ${ }^{13} \mathrm{C}$ inherent radio frequency (rf) field strength of $62.5 \mathrm{kHz}$ and ${ }^{1} \mathrm{H} \mathrm{rf}$ field strength of $127.4 \mathrm{kHz}$. During the acquisition of $27 \mathrm{~ms}$, a ${ }^{1} \mathrm{H}$ decoupling was performed with an inherent rf field of $14.7 \mathrm{kHz}$ employing WALTZ-16 sequence. In a typical $8 \mathrm{~h}$ experiment, 15,360 scans were accumulated with a recycle delay of $2.5 \mathrm{~s}$.

\section{Fourier transform infrared (FTIR) spectroscopy}

FTIR spectroscopy was conducted on Spectrum One FTIR Spectrometer (PerkinElmer, USA) at room temperature between 4000 and $600 \mathrm{~cm}^{-1}$ with a resolution of $4 \mathrm{~cm}^{-1}$. The samples were measured twice per 32 scans and average spectra were generated for each sample.

\section{Scanning electron microscopy (SEM)}

SEM images were captures by a LEO Supra-35 highresolution field emission scanning electron microscope (Carl Zeiss AG, Germany) at an accelerating voltage of $5 \mathrm{kV}$. A layer of carbon was coated on the surface of samples before SEM measurements.

\section{Polarized light micrographs (PLM)}

PLM images were obtained through Nikon ECLIPSE E600 imaging microscope with the polarizers in a perpendicular arrangement and the camera type of Nikon DS-Fi2.

\section{Laser scanning microscope (LSM)}

LSM images were obtained on a VK-X100K 3D laser scanning microscope (Keyence Corporation, Germany) under ambient conditions. 
Elemental analysis

The contents of carbon, hydrogen and sulfur were determined with an Elemental Analyser 4.1 vario EL III (Elementar, Germany). Degree of substitution (DS) of corresponding groups was calculated according to the carbon contents, as the following formula (VacaGarcia et al. 2001):

$$
\mathrm{DS}=\frac{5.13766-11.5592 \times C \%}{0.996863 \times C \%-0.856277 \times n+n \times C \%}
$$

where $C \%$ is the content of elemental carbon and $\mathrm{n}$ is the number of carbon atoms in the acyl moieties, respectively.

\section{Mechanical properties of CNW films}

The tensile testing of CNW films was performed using a Z3 micro tensile test machine equipped with a $50.0 \mathrm{~N}$ load cell from Grip-Engineering Thümler GmbH (Germany). The tensile test speed of uniaxial stretching was $2.5 \mathrm{~mm} / \mathrm{min}$. CNW films with dimensions of $20 \mathrm{~mm} \times 10 \mathrm{~mm}$ were loaded into the machine with a clamp distance of $8 \mathrm{~mm}$. For each case, three parallel tests were performed to ensure that the data collected were reliable.

\section{Results and discussion}

Preparation of CNW-SU honeycomb films

CNWs-SU with surface-immobilized 10-undecenoyl groups that we used in this study were synthesized according to our previous method by simple one-step esterification of microcrystalline cellulose with 10-undecenoyl chloride (Fig. 1a) (Liu et al. 2018). The yield was around $11 \%$ and DS ascribed to 10-undecenoyl moieties was measured to be 1.32 based on the elemental analysis. Solid-state ${ }^{13} \mathrm{C}$ CP-MAS NMR and FTIR spectra of MCC and CNWs-SU confirmed the chemical compositions (Fig. S2 and S3). The intact crystalline core was maintained within CNWs-SU according to previous analysis using solid-state ${ }^{13} \mathrm{C}$ NMR spectroscopy (Liu et al. 2018). Moreover, obtained CNWs-SU have an average length of $135 \pm 37 \mathrm{~nm}$ and an average diameter of $4 \pm 1 \mathrm{~nm}$ based on the measurements of 100 single CNWs-SU on TEM images (Fig. 1b). Owing to the existence of numerous 10-undecenoyl groups on the surface, CNWs-SU can be well dispersed in various non-polar solvents, such as toluene, THF and DCM. Similar to hydrophilic CNWs prepared by acid hydrolysis methods, the DCM suspensions of CNW-SU also displayed birefringence under crossed polarizers, as shown in the inset of Fig. 1b.

CNW-SU honeycomb films were prepared using a static breath figure approach in atmospheres with various relative humidity (Fig. S1). $10 \mathrm{~mL}$ of a DCM suspension of CNW-SU $(3 \mathrm{mg} / \mathrm{mL})$ was cast on Teflon mould and then stored in a glass desiccator with a relative humidity of $75 \%$ at room temperature. In the early stage of the breath figure process, the CNW-SU suspensions spread on the substrate homogeneously (Fig. 1c). The continuous evaporation of DCM cooled the surface of the CNW-SU suspensions and the surrounding water vapor formed small droplets and deposited on the surface of suspensions (Fig. 1d). The continuous condensation of water vapor and the parallel evaporation of DCM caused the growth and self-organization of the water droplets into ordered droplet array and forced the CNWs-SU to move closer together to form larger aggregates (Fig. 1e), thereby forming walls that will prevent coalescence and spread of the droplets. At the same time, the self-assembly of CNWs-SU occurred in this confined geometry of walls between the water droplets. The walls, composed of stacked and overlapped CNWs-SU, are highly arranged along the boundary of micropores. After complete evaporation of DCM and the water droplets, ordered honeycomb films with microscale pores on the top surfaces were obtained (Fig. 1f).

\section{Characterization of CNW-SU honeycomb films}

Figure 2a shows the photograph of a dried typical honeycomb film fabricated with CNWs-SU. The porous structured surface of the honeycomb film resulted in low transparency and high static water contact angle of $138 \pm 2^{\circ}$ (Fig. 2b). In comparison, the flat surface of the non-porous and more transparent CNW-SU film fabricated with the solvent casting method shows a much lower static water contact angle of $86 \pm 3^{\circ}$ (Fig. S4). The SEM measurement shows that the minimal diameter of approximately hexagonal pores was around $17.0 \pm 2.8 \mu \mathrm{m}$ and the width of the 

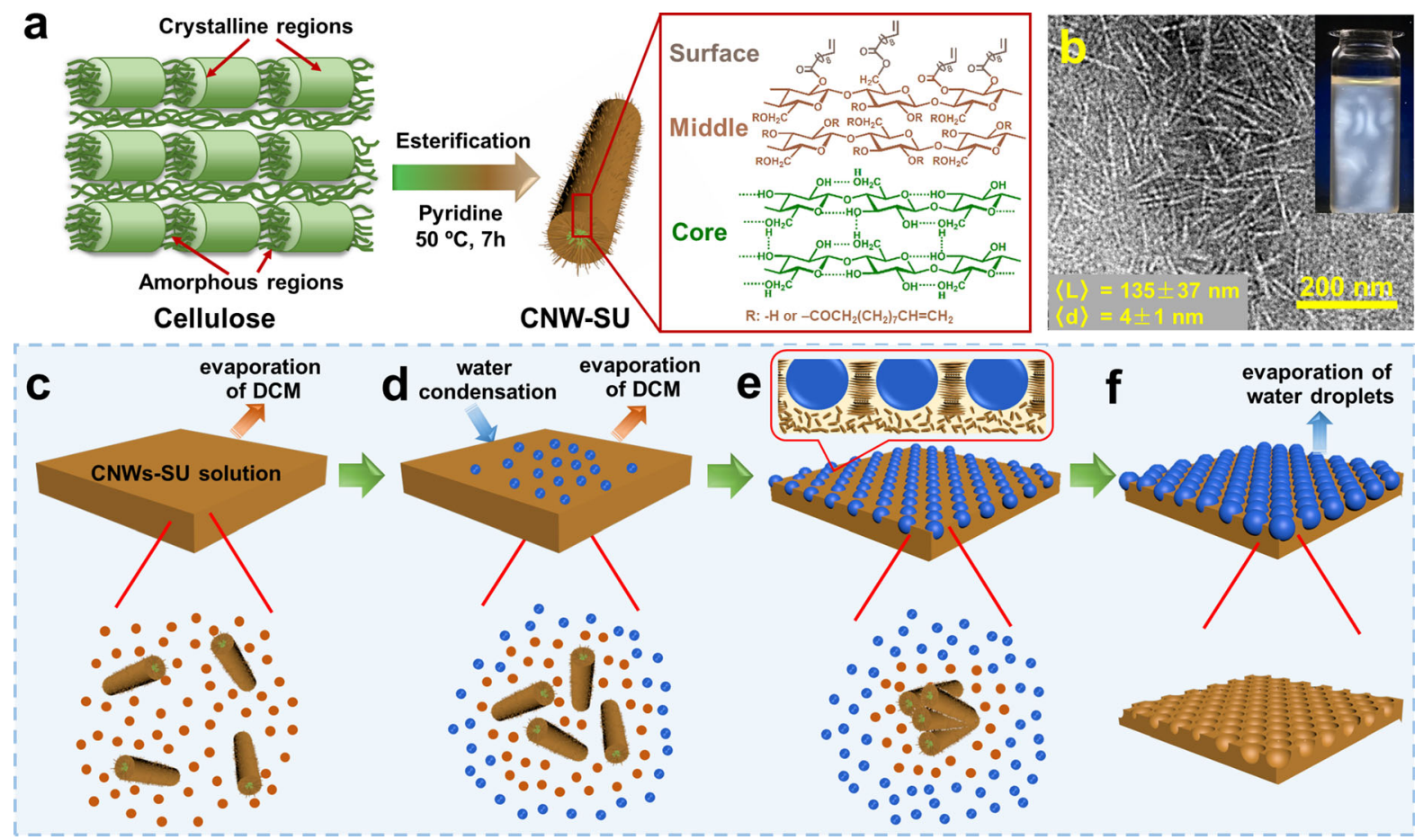

Fig. 1 a Schematic illustration for the synthesis of CNWs-SU. b A representative TEM image of CNWs-SU. The inset shows a dichloromethane suspension of CNW-SU $(15 \mathrm{mg} / \mathrm{mL})$ between

rims was about $1.8 \pm 0.4 \mu \mathrm{m}$ (Fig. 2c). The LSM measurement also confirmed the orderly-patterned honeycomb structure formed on the top surface of the films (Fig. 2d). In comparison, only a few small pores were distributed on the bottom surface of the honeycomb films (Fig. S5). Additionally, in comparison to nearly hexagonal pores of the honeycomb films in this study, the pore shape of honeycomb films fabricated with synthetic polymers through the breath figure method is generally only uniform circles ( $\mathrm{Li}$ et al. 2009; Wang et al. 2008). This could be ascribed to the fact that CNWs have high aspect ratio and high rigidity. Moreover, it is well-documented that the shape of the pores is also affected by the shape of the water droplets, which is mainly determined by the interfacial tension between the droplet and the polymer suspension, instead of the air-water interface (Ding et al. 2013). Thus, it was difficult to prepare honeycomb-patterned microporous films having regular arrays with conventional CNWs due to their high hydrophilicity.

According to the height profile obtained from 3D LSM image, the average depth of the pores crossed polarizers. c-f Schematic illustration for the selfassembly process of CNWs-SU into free-standing honeycomb films. DCM: dichloromethane

representing the height of the rims was about $3.7 \pm 0.5 \mu \mathrm{m}$ (Fig. 2e). Interestingly, the rims of obtained honeycomb films displayed iridescent colors as shown in the PLM image (Fig. 2f). Ring-shaped iridescent patterns were observed from the rims of the pores, but no iridescent color was observed from the interior part of the pores, indicating that the CNWs-SU were highly arranged along the boundary of micropores. Non-porous CNW-SU film fabricated with the same concentration of CNW-SU ( $3 \mathrm{mg} / \mathrm{mL})$ by using solvent casting method in the air under ambient conditions was used as the control and did not show any iridescence (Fig. S4). This is mainly due to the fact that the evaporation rate of DCM during the drying process for solvent cast CNW-SU films is too fast, which does not provide CNWs-SU with sufficient time for self-assembly. The SEM image of the cross-section in Fig. $2 \mathrm{~g}$ shows the contour of the pores. The depth of the pores was around $3.8 \mu \mathrm{m}$ which is consistent with the result of 3D LSM analysis. The thickness of the bottom layer was about $10.3 \pm 0.5 \mu \mathrm{m}$. Accordingly, the aspect ratio of the depth of pores and the thickness of the bottom layer $\left(\mathrm{r}_{\mathrm{D} / \mathrm{T}}\right)$ was about $35.6 \%$. 

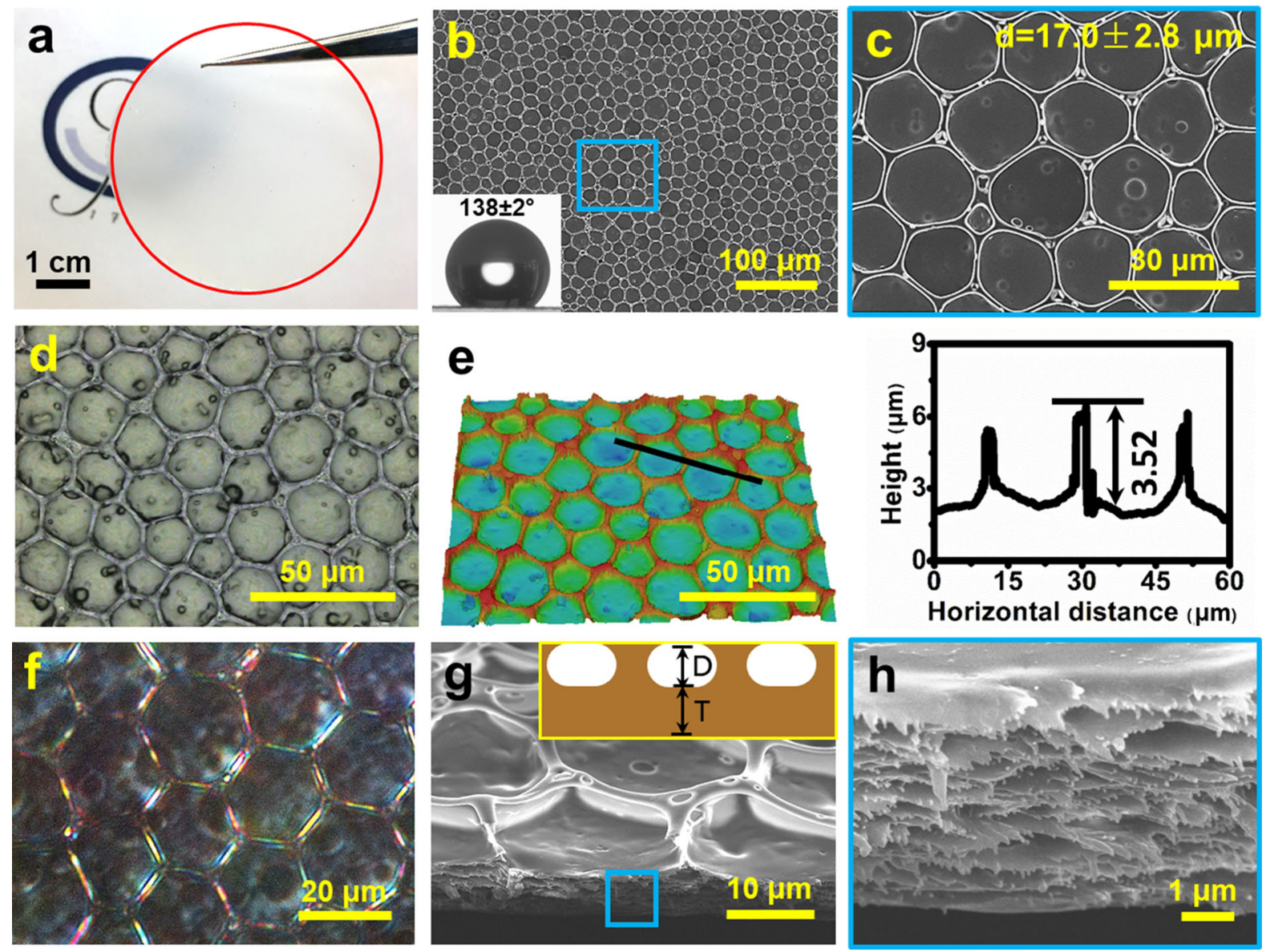

Fig. 2 a Photo image of a dried free-standing honeycomb film fabricated with CNWs-SU. b Representative SEM image of the top surface of a honeycomb film fabricated with CNWs-SU. The inset shows the static water contact angle on the top surface. c SEM image of the magnified region as in b. d Representative LSM image of a honeycomb film fabricated with CNWs-SU. e 3D LSM image and height profile of the top surface of a

Honeycomb films have unique double-layered structures supported by pillars. From the magnified SEM image of the cross section, the bottom layer of the CNW-SU films contained a typical multilayered structure (Fig. 2h). After peeling off the top layer of the top layer, by using adhesive tape, honeycomb films with a pincushion structure were obtained (Fig. 3a, $3 b)$. The obtained pinned topological features resulted in the significant increase of the static water contact angle to an average value of $144 \pm 3^{\circ}$. This is strongly coupled to the apparent surface area that is smaller than the normal close pore structure and results in lower wettability of the surface due to the formation of honeycomb film. f PLM image of a honeycomb film. g Representative SEM image for the cross section of a honeycomb film. The inset shows the schematic illustration of the calculation of $r_{D / T}$. D: the depth of pores. T: the thickness of the bottom layer. $r_{D / T}=D / T$. h SEM image of the magnified region as in $\mathbf{g}$

air pockets under the water droplet (Mongkhontreerat et al. 2015).

As well, the honeycomb films had sufficient mechanical stability (Fig. S6) and could be stretched for mechanical transformations of the shape and dimension of pores. With the assistance of applied tensile force, the micropores elongated in the direction parallel to the force, while they compressed in the perpendicular direction. Thus, the surface micropores turned from the original hexagonal shape into the elongated hexagonal shape (Fig. 3c). The ring-shaped iridescent pattern can still be obviously observed, as shown in Fig. 3d, indicating that the stretching has no 

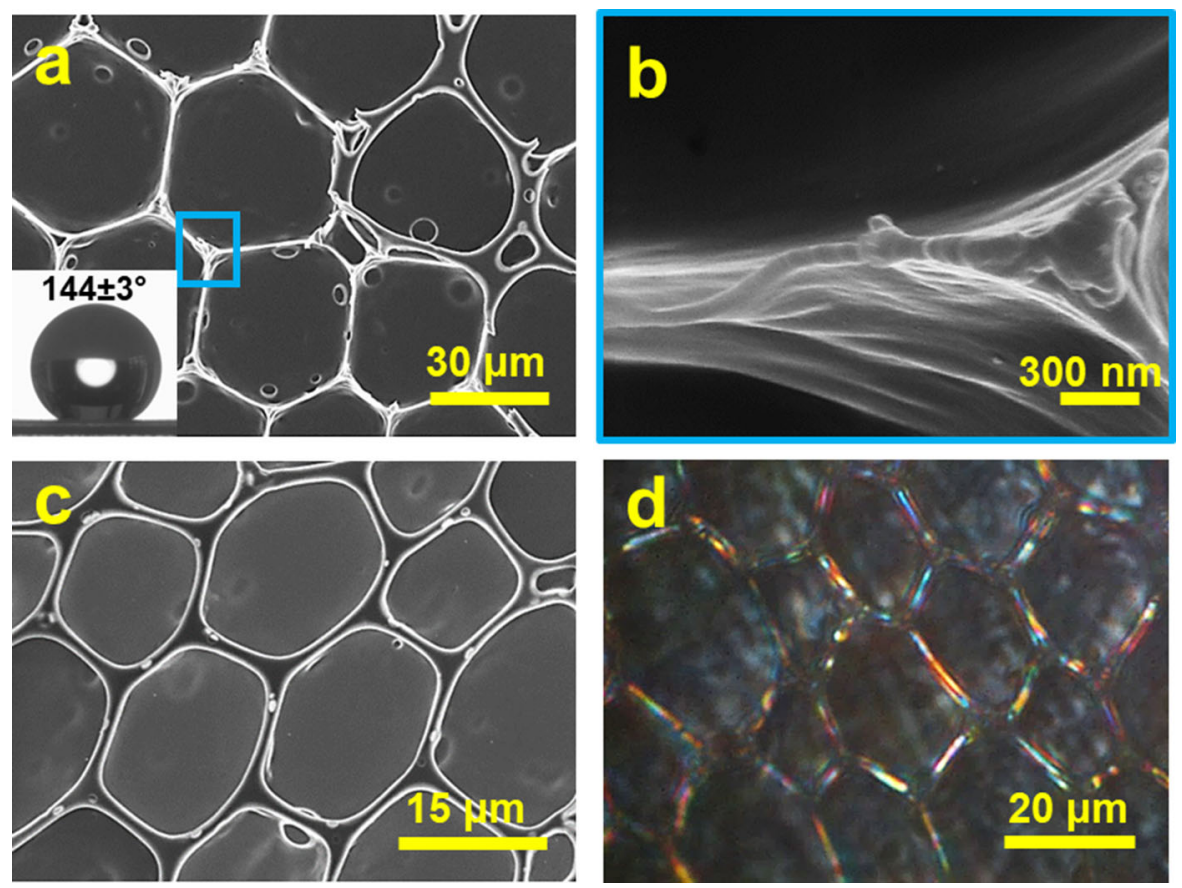

Fig. 3 a Representative SEM image of the top surface of the honeycomb film fabricated with CNWs-SU after peeling off the top layer. The inset shows static water contact angle after peeling off the top layer. $\mathbf{b}$ SEM image of the magnified region

influence on the arrangement of CNWs-SU after drying as a dried film.

\section{Mechanism of the self-assembly of CNWs-SU} into honeycomb films with iridescent rims

To understand the mechanism of the self-assembly of CNWs-SU into honeycomb films with iridescent rims, pore walls of the films were further characterized. As shown in the SEM images of the damaged pores, the cross section of the pore walls presents a ginkgo-leaflike shape (Fig. 4a, b). Additionally, the layered structure with a periodic spacing of about $300 \mathrm{~nm}$ was observed (Fig. 4b). Moreover, the bottom side of the rims contains a slanted layered structure (Fig. 4c, d). This is mainly because the width of the rims is getting narrower from bottom to top. During the selfassembly process, the water droplets grew slowly, while CNWs-SU were squeezed and formed the slanted layered structure within the confined space between the droplets. The mechanism of the iridescent rim formation during the generation of honeycomb films by the breath figure method was also proposed

as in a. c Representative SEM image the dried honeycomb film fabricated with CNWs-SU after stretching. d PLM image the dried honeycomb film fabricated with CNWs-SU after stretching

(Fig. 4e). The CNW-SU concentration was uniform in the initial aqueous suspension. As the water droplets formed on the surface of the suspension, the DCM evaporated from the areas where no water droplets covered. With the evaporation of DCM, the concentration of CNW-SU in the confined 3D walls becomes gradually higher. After the total evaporation of DCM in the confined 3D walls and water, CNWs-SU selfassembled into cholesteric architectures and resulted in iridescent colors of the rims. The arrangement presumably occurs upon the growth of the aqueous droplets during the breath figure process, which determines the optical properties of the resulting films.

During the formation of honeycomb films, their morphologies were found to be strongly affected by a few parameters. Among others are the concentration of CNW-SU in their suspensions, the relative humidity in the surroundings and the surface-attached moieties that were further introduced onto the terminal vinyl groups via thiol-ene reactions.

The pore size and regularity of breath figure arrays are sensitive to the concentration of the suspensions. As a result, each suspension of CNW-SU with a 

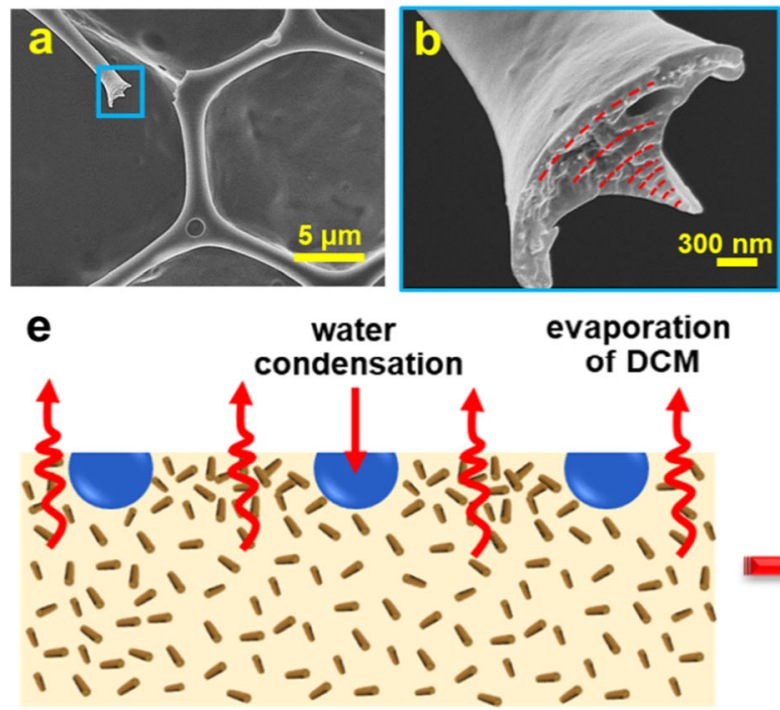

Fig. 4 a Representative SEM image of the rims in a honeycomb film fabricated with CNWs-SU. b SEM image of the magnified region as in a. $\mathbf{c}$ Representative SEM image of the cross section

different concentration yielded an entirely different morphology. Using CNW-SU suspensions with the concentration of $1.5 \mathrm{mg} / \mathrm{mL}$, only a few small pores were randomly distributed on the top surface of obtained films (Fig. 5a), indicating that an adequate amount of CNW-SU is a prerequisite for the formation of highly ordered honeycomb films. This may stem from the insufficient encapsulation and stabilization of the condensed water droplets on the interface due to the low quantity of CNW-SU. When the concentration of CNW-SU increased from $3 \mathrm{mg} / \mathrm{mL}$ to $7 \mathrm{mg} / \mathrm{mL}$, a honeycomb-structured, porous film with a higher average pore size of $22.9 \pm 2.9 \mu \mathrm{m}$ was also obtained and the depth of the pores increased from $3.7 \pm 0.5 \mu \mathrm{m}$ to $7.5 \pm 1.0 \mu \mathrm{m}$ according to the result of LSM (Fig. 5c, S7a, S7b, and S7c). Moreover, the resulting films exhibited pores with irregular shapes. It is therefore evident that the increase of the concentration facilitates the formation of deeper holes and thus higher rims. The thickness of the bottom layer was about $18.0 \pm 0.7 \mu \mathrm{m}$. Accordingly, the $\mathrm{r}_{\mathrm{D} / \mathrm{T}}$ was about $41.7 \%$, which was higher than the honeycomb film fabricated at the concentration of $3 \mathrm{mg} / \mathrm{mL}(35.6 \%)$. On the other hand, as shown in Fig. S7d, no obvious iridescent color of the rim was observed and thus it can be concluded that the high concentration is not beneficial to the self-assembly of CNWs-SU in such geometry. A further increase in the concentration of
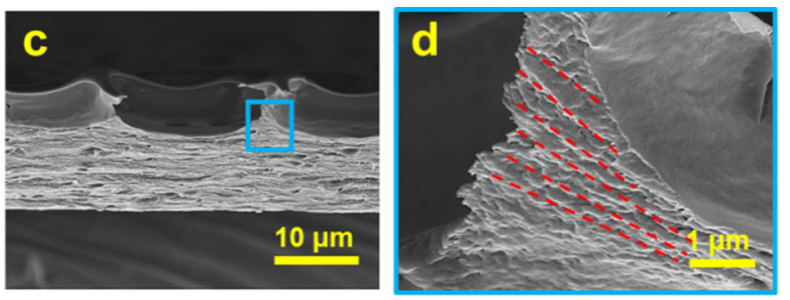

\section{evaporation of DCM}

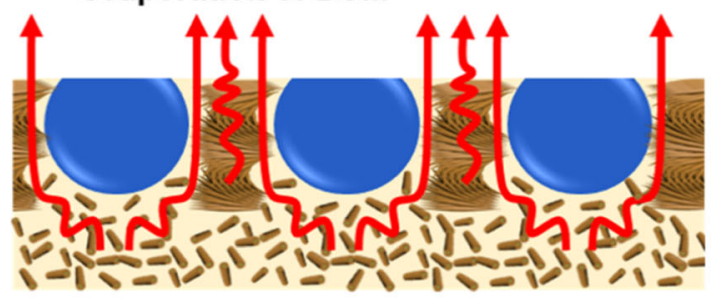

of broken rims of a honeycomb film. d SEM image of the magnified region as in c. e Schematic description for the formation of the iridescent rims

CNW-SU suspension to $16 \mathrm{mg} / \mathrm{mL}$ resulted in films with few pores of irregular morphology (Fig. 5d). This also verifies that $\mathrm{CNW}-\mathrm{SU}$ with a too high concentration cannot effectively self-assemble into highly ordered structures in this 3D confinement. The increased viscosity should have weakened the convection in the suspension, which is not beneficial to the arrangement of water droplets. At the same time, the pores will become disordered when the concentration is too high (Zhao et al. 2006).

The humidity of the atmosphere as another key parameter for the self-assembly process of CNWs-SU also influences the water condensation at the airsuspension interface. When the relative humidity was below $50 \%$, only flat films with an irregular arrangement of a few pores were obtained (Fig. 5e). This was because water vapor cannot effectively condensate into micrometer-sized droplets for templating the generation of pores under such humidity. When the relative humidity increased to $60 \%$, many micropores were unevenly distributed on the top surface of the films, although some small areas without pores can still be found (Fig. 5f). The average pore size was about $12.9 \pm 1.7 \mu \mathrm{m}$ and the depth of pores equal to the height of rims was about $3.2 \pm 0.9 \mu \mathrm{m}$ (Fig. $5 f$ and Fig. S8). As the relative humidity of the atmosphere increased from 75 to $96 \%$, the average pore size increased positively from $17.0 \pm 2.8$ to 


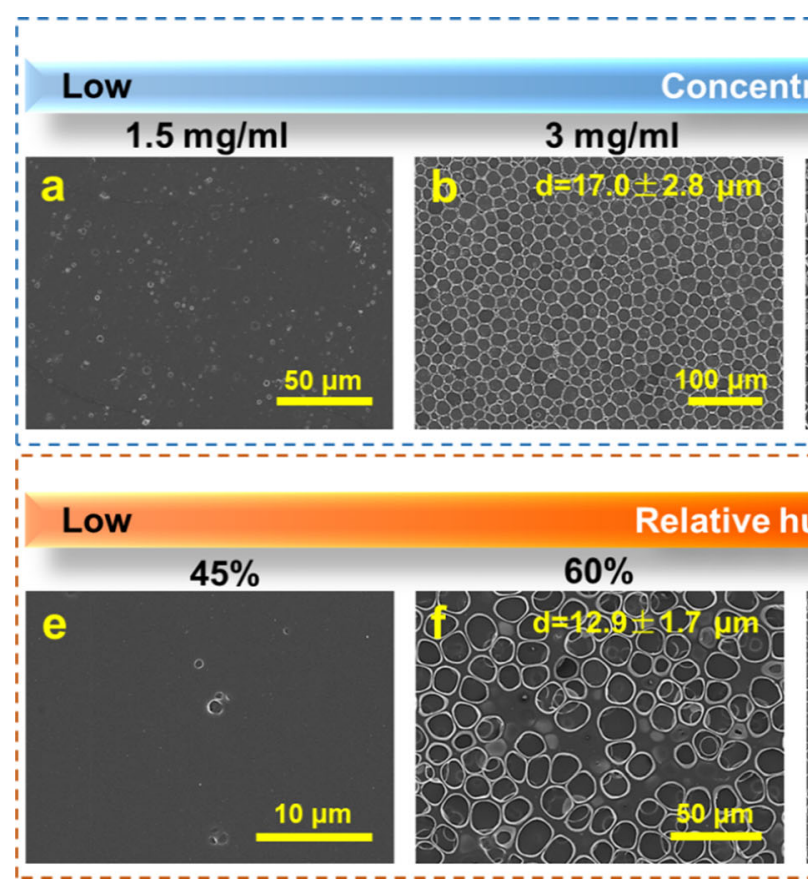

Fig. 5 Formation mechanism of the honeycomb films by CNWs-SU. Representative SEM image of the top surface of honeycomb films fabricated with CNWs-SU at the relative humidity of $75 \%$ with the concentration of a $1.5 \mathrm{mg} / \mathrm{mL}$,

$24.3 \pm 3.8 \mu \mathrm{m}$ (Fig. 5g, h). The high relative humidity results in the coalescence of water droplets, yielding a polydisperse pore size distribution. The depth of pores increased from $3.7 \pm 0.5$ to $10.6 \pm 2.2 \mu \mathrm{m}$ with increasing the relative humidity from 75 to $96 \%$ (Fig. S9), while the thickness of the bottom layer maintained almost unchanged. Thus, the $\mathrm{r}_{\mathrm{D} / \mathrm{T}}$ was about $98.1 \%$, which was much higher than the honeycomb film fabricated at the relative humidity of $75 \%$. Therefore, higher relative humidity not only facilitates the formation of larger water droplets but also slows the solvent evaporation, both of which lead to the growth of droplets and the formation of larger pores (Wang et al. 2008). The relative humidity of the atmosphere influences the evaporation of the CNWSU solvents, which further affects the self-assembly of CNWs-SU in the confined 3D geometry. This is also reflected in the unobvious iridescent colors of the rims (Fig. S8d, S9d).

To further investigate the effect of the surfaceattached moieties of CNWs on the pore morphology, the CNWs-SU were further modified with octadecane or fluoroalkyl moieties (75\% of the total vinyl groups) b $3 \mathrm{mg} / \mathrm{mL}$, c $7 \mathrm{mg} / \mathrm{mL}$, and d $16 \mathrm{mg} / \mathrm{mL}$. Representative SEM image of the top surface of honeycomb films fabricated with CNWs-SU with the concentration of $3 \mathrm{mg} / \mathrm{mL}$ at the relative humidity of e $45 \%$, f $60 \%, \mathbf{g} 75 \%$, and h $96 \%$

onto the vinyl groups. The resultant CNWs, namely CNWs-SU-C18 and CNWs-SU-F, were also used to prepare honeycomb films. Although films with porous surface structure were successfully fabricated with both CNWs-SU-C18 and CNWs-SU-F, the obtained CNW-SU-C18 and CNW-SU-F films had lower transparency (Fig. 6a, d) and displayed distinct pore morphologies compared with CNW-SU films. The average pore size of the films fabricated from CNWsSU-C18 was $49.2 \pm 5.8 \mu \mathrm{m}$, which was much larger than that of the film fabricated with CNWs-SU. Moreover, it can be seen from Fig. $6 b$ and $6 c$ that pores with the diameter of dozens of micros as well as relatively small pores of several micros were formed on the surface of CNW-SU-C18 films. In addition, the small pores were distributed randomly around the large pores.

Compared with the CNW-SU films and CNW-SUC18 films, CNW-SU-F films displayed porous surface structure with more irregular-shaped pores of larger sizes (Fig. 6e, f). The average pore size of the films fabricated from CNWs-SU-F was about $70.3 \pm 13.1 \mu \mathrm{m}$. The CNWs-SU with surface- 

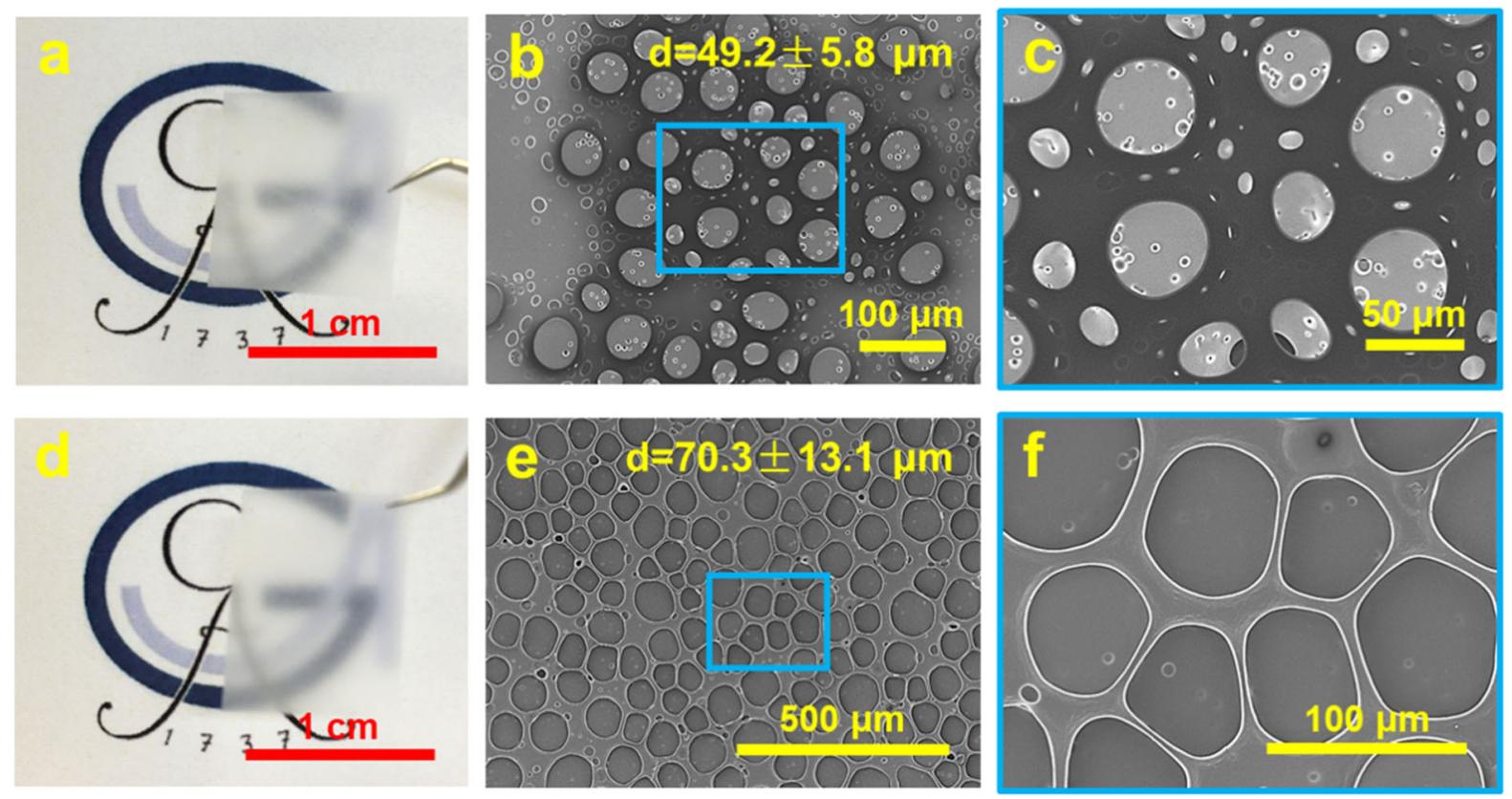

Fig. 6 a Photo image of a dried free-standing porous film fabricated with CNWs-SU-C18. b Representative SEM image of the top surface of a dried free-standing porous film fabricated with CNWs-SU-C18 at the relative humidity of $75 \%$ with a concentration of $3 \mathrm{mg} / \mathrm{mL}$. c SEM images of the magnified

attached octadecane or fluoroalkyl groups had higher hydrophobicity and did not stabilize the condensed water droplets effectively, leading to the coalescing of water droplets. The random coalescence of water droplets resulted in the formation of larger pores with irregular shapes. Thus, it can be concluded that the surface-immobilized functional groups of CNWs also have great effect on the morphology of the resultant films fabricated by the breath figure method. In addition, no iridescence was observed from the rim of the CNW-SU-C18 and CNW-SU-F films, which indicates an altered coalescence process during drying (Fig. S10).

\section{Conclusions}

In summary, porous honeycomb-patterned free-standing films were prepared with CNWs-SU by using the breath figure method. The rims were composed of selfassembled and stacked CNWs-SU, which are highly arranged along the boundary of microsized pores. Owing to the ordered structures formed by the selfassembly of CNWs-SU in the confined 3D geometry, region as in $\mathbf{b}$. d Photo image of a dried free-standing porous film fabricated with CNWs-SU-F. e Representative SEM image of the top surface of a porous film fabricated with CNWs-SU-F at the relative humidity of $75 \%$ with a concentration of $3 \mathrm{mg} /$ $\mathrm{mL}$. f SEM images of the magnified region as in $\mathbf{e}$

the rims of the obtained honeycomb films displayed iridescent colors. The arrangement of CNWs-SU presumably occurs upon the growth of the aqueous droplets during the breath figure process. A few critical factors, primarily the concentration of the CNW-SU suspension, the relative humidity of the atmosphere and surface-attached moieties, affected the self-assembly process. This self-assembly of hydrophobic CNWs-SU within constrained space unlocks new possibilities in designing the visual appearance of such iridescent films. We expect these porous films with structural colors will find a broad range of applications, such as for filtration or sophisticated optical materials.

Acknowledgments K.Z. thanks German Research Foundation (DFG) for the financial support for the project with the project Nr. ZH546/2-1 and Georg-August-University of Goettingen for the Department start-up funding. H.L. and B.P. thank the China Scholarship Council (CSC) for the financial support.

Funding Open Access funding enabled and organized by Projekt DEAL. 
Data availability All the data and materials are accessible.

\section{Declarations}

Conflict of interest The authors declare that they have no conflict of interest.

Open Access This article is licensed under a Creative Commons Attribution 4.0 International License, which permits use, sharing, adaptation, distribution and reproduction in any medium or format, as long as you give appropriate credit to the original author(s) and the source, provide a link to the Creative Commons licence, and indicate if changes were made. The images or other third party material in this article are included in the article's Creative Commons licence, unless indicated otherwise in a credit line to the material. If material is not included in the article's Creative Commons licence and your intended use is not permitted by statutory regulation or exceeds the permitted use, you will need to obtain permission directly from the copyright holder. To view a copy of this licence, visit http://creativecommons.org/licenses/by/4.0/.

\section{References}

Adstedt K, Popenov EA, Pierce KJ, Xiong R, Geryak R, Cherpak V, Nepal D, Bunning TJ, Tsukruk VV (2020) Chiral cellulose nanocrystals with intercalated amorphous polysaccharides for controlled iridescence and enhanced mechanics. Adv Funct Mater 30:2003597. https://doi.org/ 10.1002/adfm.202003597

Bai H, Du C, Zhang A, Li L (2013) Breath figure arrays: Unconventional fabrications, functionalizations, and applications. Angew Chem Int Ed 52:12240-12255. https://doi.org/10.1002/anie.201303594

Bondeson D, Mathew A, Oksman K (2006) Optimization of the isolation of nanocrystals from microcrystalline celluloseby acid hydrolysis. Cellulose 13:171-180. https://doi.org/10. 1007/s10570-006-9061-4

Bui V-T, Ko SH, Choi H-S (2015) Large-scale fabrication of commercially available, nonpolar linear polymer film with a highly ordered honeycomb pattern. ACS Appl Mater Interf 7:10541-10547. https://doi.org/10.1021/acsami. $5 \mathrm{~b} 02097$

Bunz UHF (2006) Breath figures as a dynamic templating method for polymers and nanomaterials. Adv Mater 18:973-989. https://doi.org/10.1002/adma.200501131

Cherpak V, Korolovych VF, Geryak R, Turiv T, Nepal D, Kelly J, Bunning TJ, Lavrentovich OD, Heller WT, Tsukruk VV (2018) Robust chiral organization of cellulose nanocrystals in capillary confinement. Nano Lett 18:6770-6777. https:// doi.org/10.1021/acs.nanolett.8b02522

Ding J, Zhang A, Bai H, Li L, Li J, Ma Z (2013) Breath figure in non-aqueous vapor. Soft Matter 9:506-514. https://doi.org/ 10.1039/C2SM27093E

Du C, Zhang A, Bai H, Li L (2013) Robust microsieves with excellent solvent resistance: cross-linkage of perforated polymer films with honeycomb structure. ACS Macro Lett 2:27-30. https://doi.org/10.1021/mz300616z

Dumanli AG, van der Kooij HM, Kamita G, Reisner E, Baumberg JJ, Steiner U, Vignolini S (2014) Digital color in cellulose nanocrystal films. ACS Appl Mater Interf 6:12302-12306. https://doi.org/10.1021/am501995e

Galeotti F, Mróz W, Bolognesi A (2011) CdTe nanocrystal assemblies guided by breath figure templates. Soft Matter 7:3832-3836. https://doi.org/10.1039/C1SM05148B

Giese M, Spengler M (2019) Cellulose nanocrystals in nanoarchitectonics-towards photonic functional materials. Mol Syst Des Eng 4:29-48. https://doi.org/10.1039/ C8ME00065D

Habibi Y, Lucia LA, Rojas OJ (2010) Cellulose nanocrystals: chemistry, self-assembly, and applications. Chem Rev 110:3479-3500. https://doi.org/10.1021/cr900339w

Hiratani T, Hamad WY, MacLachlan MJ (2017) Transparent depolarizing organic and inorganic films for optics and sensors. Adv Mater 29:1606083. https://doi.org/10.1002/ adma.201606083

Hu J, Abdelsalam M, Bartlett P, Cole R, Sugawara Y, Baumberg J, Mahajan S, Denuault G (2009) Electrodeposition of highly ordered macroporous iridium oxide through selfassembled colloidal templates. J Mater Chem 19:3855-3858. https://doi.org/10.1039/B900279K

Huang J, Zhu J, Sun W, Ji J (2020) Versatile and functional surface patterning of in situ breath figure pore formation via solvent treatment. ACS Appl Mater Interf 12:47048-47058. https://doi.org/10.1021/acsami.0c14614

Ju Y, Ding L, Zhu J, Sun W (2019) Fabrication of honeycombstructured protein arrays via one-step method. J Appl Polym Sci 136:47084. https://doi.org/10.1002/app.47084

Kasai W, Kondo T (2004) Fabrication of honeycomb-patterned cellulose films. Macromol Biosci 4:17-21. https://doi.org/ 10.1002/mabi.200300054

Kim JH, Seo M, Kim SY (2009) Lithographically patterned breath figure of photoresponsive small molecules: dualpatterned honeycomb lines from a combination of bottomup and top-down lithography. Adv Mater 21:4130-4133. https://doi.org/10.1002/adma.200900868

Lee SH, Kim HW, Hwang JO, Lee WJ, Kwon J, Bielawski CW, Ruoff RS, Kim SO (2010) Three-dimensional self-assembly of graphene oxide platelets into mechanically flexible macroporous carbon films. Angew Chem Int Ed 49:10084-10088. https://doi.org/10.1002/ange.201006240

Li L, Zhong Y, Li J, Chen C, Zhang A, Xu J, Ma Z (2009) Thermally stable and solvent resistant honeycomb structured polystyrene films via photochemical cross-linking. J Mater Chem 19:7222-7227. https://doi.org/10.1039/ B911714H

Li Y, Jun-Yan Suen J, Prince E, Larin EM, Klinkova A, ThérienAubin H, Zhu S, Yang B, Helmy AS, Lavrentovich OD, Kumacheva E (2016) Colloidal cholesteric liquid crystal in spherical confinement. Nat Commun 7:12520. https://doi. org/10.1038/ncomms 12520

Liu Y, Wu P (2020) Bioinspired hierarchical liquid-metacrystal fibers for chiral optics and advanced textiles. Adv Funct Mater 30:2002193. https://doi.org/10.1002/adfm. 202002193

Liu H, Pang B, Garces R, Dervisoglu R, Chen L, Andreas L, Zhang K (2018) Helical fibers via evaporation-driven self- 
assembly of surface-acylated cellulose nanowhiskers. Angew Chem Int Ed 57:16323-16328. https://doi.org/10. 1002/ange.201808250

Mahpeykar SM, Zhao Y, Li X, Yang Z, Xu Q, Lu ZH, Sargent EH, Wang X (2017) Cellulose nanocrystal: Polymer hybrid optical diffusers for index-matching-free light management in optoelectronic devices. Adv Opt Mater 5:1700430. https://doi.org/10.1002/adom.201700430

Min E, Wong KH, Stenzel MH (2008) Microwells with patterned proteins by a self-assembly process using honeycomb-structured porous films. Adv Mater 20:3550-3556. https://doi.org/10.1002/adma.200800569

Mongkhontreerat S, Walter MV, Andrén OCJ, Cai Y, Malkoch M (2015) Beyond state of the art honeycomb membranes: high performance ordered arrays from multiprogrammable linear-dendritic block copolymers. Adv Funct Mater 25:4837-4843. https://doi.org/10.1002/adfm.201501643

Nemoto J, Uraki Y, Kishimoto T, Sano Y, Funada R, Obata N, Yabu H, Tanaka M, Shimomura M (2005) Production of mesoscopically patterned cellulose film. Bioresour Technol 96:1955-1958. https://doi.org/10.1016/j.biortech. 2005.01.034

Neznalová K, Fajstavr D, Rimpelová S, Kasálková NS, Kolská Z, Švorčík V, Slepička P (2020) Honeycomb-patterned poly(L-lactic) acid on plasma-activated FEP as cell culture scaffold. Polym Degrad Stab 181:109370. https://doi.org/ 10.1016/j.polymdegradstab.2020.109370

Parker RM, Frka-Petesic B, Guidetti G, Kamita G, Consani G, Abell C, Vignolini S (2016) Hierarchical self-assembly of cellulose nanocrystals in a confined geometry. ACS Nano 10:8443-8449. https://doi.org/10.1021/acsnano.6b03355

Sakatani Y, Boissière C, Grosso D, Nicole L, Soler-Illia GJAA, Sanchez C (2008) Coupling nanobuilding block and breath figures approaches for the designed construction of hierarchically templated porous materials and membranes. Chem Mater 20:1049-1056. https://doi.org/10.1021/ cm701986b

Slepička P, Neznalová K, Fajstavr D, Švorčík V (2020) Nanostructuring of honeycomb-like polystyrene with excimer laser. Prog Org Coat 145:105670. https://doi.org/ 10.1016/j.porgcoat.2020.105670

Vaca-Garcia C, Borredon ME, Gaseta A (2001) Determination of the degree of substitution (DS) of mixed cellulose esters by elemental analysis. Cellulose 8:225-231. https://doi. org/10.1023/A:1013133921626

Wakamatsu N, Takamori H, Fujigaya T, Nakashima N (2009) Self-organized single-walled carbon nanotube conducting thin films with honeycomb structures on flexible plastic films. Adv Funct Mater 19:311-316. https://doi.org/10. 1002/adfm.200801241

Wan LS, Li QL, Chen PC, Xu ZK (2012) Patterned biocatalytic films via one-step self-assembly. Chem Commun 48:4417-4419. https://doi.org/10.1039/C2CC17451K

Wang C, Mao Y, Wang D, Qu Q, Yang G, Hu X (2008) Fabrication of highly ordered microporous thin films by PS-b-PAA self-assembly and investigation of their tunable surface properties. J Mater Chem 18:683-690. https://doi. org/10.1039/B715520D

Yabu H, Shimomura M (2005) Simple fabrication of micro lens arrays. Langmuir 21:1709-1711. https://doi.org/10.1021/ la046996z

Yao K, Meng Q, Bulone V, Zhou Q (2017) Flexible and responsive chiral nematic cellulose nanocrystal/poly(ethylene glycol) composite films with uniform and tunable structural color. Adv Mater 29:1701323. https://doi.org/10. 1002/adma.201701323

Young JF (1967) Humidity control in the laboratory using salt solutions-a review. J Appl Chem 17:241-245. https://doi. org/10.1002/jctb.5010170901

Zhang A, Bai H, Li L (2015) Breath figure: a nature-inspired preparation method for ordered porous films. Chem Rev 115:9801-9868. https://doi.org/10.1021/acs.chemrev. 5b00069

Zhang X, Xiong R, Kang S, Yang Y, Tsukruk VV (2020) Alternating stacking of nanocrystals and nanofibers into ultrastrong chiral biocomposite laminates. ACS Nano 14:14675-14685. https://doi.org/10.1021/acsnano. $0 \mathrm{c} 06192$

Zhao B, Zhang J, Wang X, Li C (2006) Water-assisted fabrication of honeycomb structure porous film from poly(1lactide). J Mater Chem 16:509-513. https://doi.org/10. 1039/B512398D

Publisher's Note Springer Nature remains neutral with regard to jurisdictional claims in published maps and institutional affiliations. 